TRANSACTIONS OF THE

AMERICAN MATHEMATICAL SOCIETY

Volume 179, May 1973

\title{
SUMMABILITY OF JACOBI SERIES
}

\author{
BY \\ RICHARD ASKEY(1)
}

\begin{abstract}
The positivity of some Cesàro mean is proven for Jacobi series $\sum a_{n} P_{n}^{(\alpha, \beta)}(x), \alpha, \beta \geqq-\frac{1}{2}$. This has applications to the mean convergence of Lagrange interpolation at the zeros of Jacobi polynomials. The positivity of the $(C, \alpha+\beta+2)$ means is conjectured and proven for some $(\alpha, \beta)$. One consequence of this conjecture would be the complete monotonicity of $x^{-c}\left(x^{2}+1\right)^{-c}, c \geqq 1$.
\end{abstract}

1. Introduction. Summability of series is an old subject which has fallen into disrepute in most mathematical circles. However some interesting problems still exist for summability of orthogonal series of Jacobi polynomials. We will obtain some new results, which generalize classical results of Fejér [11], Kogbetliantz [17], and a relatively recent result of Fejér [13]. The 1933 date of this paper gives the reader an idea when this subject was last considered. Certain new results for Hankel transforms follow from these Jacobi series results and a number of open problems arise. The easiest to explain is:

If

$$
\frac{1}{x^{c}\left(x^{2}+1\right)^{c}}=\int_{0}^{\infty} K_{c}(t) e^{-x t} d t,
$$

show that $K_{c}(t) \geqq 0, c \geqq 1$.

This is easy for $c=1,2, \ldots$, and is probably false for $c<1$. It fails for $c=\frac{1}{2}$.

My interest in Cesàro summability of Jacobi series stems from work on $L^{p}$ convergence of Lagrange interpolation at the zeros of Jacobi polynomials [1]. The only missing step in the proof of $L^{p}$ convergence of Lagrange interpolation polynomials at the zeros of fairly general orthogonal polynomials is the positivity of the Cesàro means of some order. This will be proven for most Jacobi series.

The first hard result was found by Kogbetliantz when he proved the positivity of the $(C, 2 \alpha+2)$ means of

$$
P_{r}(x, y)=\sum_{n=0}^{\infty} r^{n} P_{n}^{(\alpha, \beta)}(x) P_{n}^{(\alpha, \beta)}(y) / \int_{-1}^{1}\left[P_{n}^{(\alpha, \beta)}(x)\right]^{2}(1-x)^{\alpha}(1+x)^{\beta} d x
$$

for $\alpha=\beta \geqq-\frac{1}{2}$.

Presented to the Society, November 20, 1971; received by the editors May 2, 1972.

AMS (MOS) subject classifications (1970). Primary 33A65, 40G05, 42A56; Secondary 33A40, 41A05.

Key words and phrases. Positive Cesàro sum, Jacobi polynomial, Bessel function, Poisson kernel, delayed means, Lagrange interpolation.

(1) Supported in part by NSF Grant GP-24182 and the United States Army under contract number DA-31-124-ARO-D-462. 
I have never felt I understood this result. It should be compared with the following result of Szegö [24, Theorem 9.1.3].

If $f(x)$ is continuous on $[-1,1]$ then the Jacobi series

$$
f(x) \sim \sum_{n=0}^{\infty} a_{n} P_{n}^{(\alpha, \beta)}(x), \quad \alpha \geqq \beta \geqq-\frac{1}{2},
$$

is uniformly $\left(C, \alpha+\frac{1}{2}+\varepsilon\right)$ summable, $\varepsilon>0$. There is a continuous function which is not $\left(C, \alpha+\frac{1}{2}\right)$ summable at $x=1$.

Actually Szegö only proves the $\left(C, \alpha+\frac{1}{2}+\varepsilon\right)$ summability at $x=1$, but the convolution structure for Jacobi series [6], [15] (which will be described below) gives the uniform summability. The point that bothered me was the rate of growth of these two functions in $\alpha$. Why should Szegö's result grow like one times $\alpha$ while Kogbetliantz's result grows like two times $\alpha$ ? The clue is provided by a little known result of Fejér [13]. He proved the positivity of the $(C, 2)$ means of

$$
\sum_{n=1}^{\infty}(2 n-1) \sin (2 n-1) \theta \text {. }
$$

This is the $(C, 2)$ summability of $(1.1)$ for $(\alpha, \beta)=\left(\frac{1}{2},-\frac{1}{2}\right)$. Fejér also observed that the $(C, 2)$ summability of (1.1) for $\alpha=\beta=0$ follows from this result. $\mathrm{He}$ used an integral of Mehler to prove this. A generalization of Mehler's integral due to Bateman can be used to show the positivity of the $(C, 2)$ means of $(1.1)$ for $\alpha+\beta=0,0<\alpha<\frac{1}{2}$. This suggests that the $(C, \alpha+\beta+2)$ means of (1.1) are positive for $\alpha, \beta \geqq-\frac{1}{2}$. This will be shown for $\alpha=\beta+1, \beta \geqq-\frac{1}{2}$, and some other values. We will also show that it holds for $(\alpha-\mu, \beta+\mu), 0<\mu$ $<(\alpha-\beta) / 2$, if it holds for $(\alpha, \beta)$. These results will be given in $\$ 3$. $\$ 2$ will contain the necessary preliminaries on Poisson summability. The corresponding results and problems for Hankel transforms will be in $\$ 4$. Applications will be given in $\$ 5$.

2. Poisson kernel. Before introducing the Cesàro means we must calculate the Poisson kernel for Jacobi series. This is

$$
\begin{aligned}
P_{r}(x, y)=\sum_{n=0}^{\infty} r^{n} P_{n}^{(\alpha, \beta)}(x) P_{n}^{(\alpha, \beta)}(y) / \int_{-1}^{1}\left[P_{n}^{(\alpha, \beta)}(x)\right]^{2}(1-x)^{\alpha}(1+x)^{\beta} d x & \\
\alpha, \beta & >-1 .
\end{aligned}
$$

(2.1) was computed by Bailey [7] and the positivity of $P_{r}(x, y)$ for $0 \leqq r<1$, $-1 \leqq x, y \leqq 1$ is obvious from his formula. However his formula is a double sum and so is very complicated to handle. For $\alpha, \beta \geqq-\frac{1}{2}$ or $\alpha+\beta \geqq 0$ there is another way to proceed. By symmetry,

$$
P_{n}^{(\alpha, \beta)}(-x)=(-1)^{n} P_{n}^{(\beta, \alpha)}(x), \quad[24,(4.13)]
$$

we may assume $\alpha \geqq \beta$. Using a recent result of Gasper [15], [16] it is sufficient to consider $y=1$. Gasper's result is 
Theorem A. If $\int_{-1}^{1}|f(x)|(1-x)^{\alpha}(1+x)^{\beta} d x<\infty, f(x)$ is measurable, $\alpha \geqq \beta$ $>-1$ and either $\beta \geqq-\frac{1}{2}$ or $\alpha+\beta \geqq 0$, and

$$
a_{n}=\int_{-1}^{1} f(x) P_{n}^{(\alpha, \beta)}(x)(1-x)^{\alpha}(1+x)^{\beta} d x,
$$

then there is a function $f(x, y)$ measurable in $(x, y)$,

$$
\int_{-1}^{1}|f(x, y)|(1-x)^{\alpha}(1+x)^{\beta} d x<\infty
$$

for which

$$
a_{n} \frac{P_{n}^{(\alpha, \beta)}(y)}{P_{n}^{(\alpha, \beta)}(1)}=\int_{-1}^{1} f(x, y) P_{n}^{(\alpha, \beta)}(x)(1-x)^{\alpha}(1+x)^{\beta} d x
$$

i.e.

$$
f(x, y) \sim \sum_{n=0}^{\infty} a_{n} h_{n}^{(\alpha, \beta)} P_{n}^{(\alpha, \beta)}(x) P_{n}^{(\alpha, \beta)}(y) / P_{n}^{(\alpha, \beta)}(1), \quad f(x, 1)=f(x)
$$

and

$$
f(x) \sim \sum_{n=0}^{\infty} a_{n} h_{n}^{(\alpha, \beta)} P_{n}^{(\alpha, \beta)}(x)
$$

where

$$
\begin{aligned}
{\left[h_{n}^{\alpha, \beta}\right] } & =\left[\int_{-1}^{1}\left[P_{n}^{(\alpha, \beta)}(x)\right]^{2}(1-x)^{\alpha}(1+x)^{\beta} d x\right]^{-1} \\
& =\frac{(2 n+\alpha+\beta+1) \Gamma(n+\alpha+\beta+1) n !}{2^{\alpha+\beta+1} \Gamma(n+\alpha+1) \Gamma(n+\beta+1)} .
\end{aligned}
$$

If $f(x) \geqq 0$ then $f(x, y) \geqq 0$.

Another proof of the case $\alpha \geqq \beta \geqq-\frac{1}{2}$ is given in [18].

This last positivity result is crucial. When $y=1, P_{r}(x, y)$ is

$$
\begin{aligned}
P_{r}(x, 1) & =P_{r}(x) \\
& =\sum_{n=0}^{\infty} \frac{(2 n+\alpha+\beta+1) \Gamma(n+\alpha+\beta+1)}{2^{\alpha+\beta+1} \Gamma(n+\beta+1) \Gamma(\alpha+1)} P_{n}^{(\alpha, \beta)}(x) r^{n},
\end{aligned}
$$

since

$$
P_{n}^{(\alpha, \beta)}(1)=(\alpha+1)_{n} / n !=\Gamma(n+\alpha+1) /[\Gamma(\alpha+1) n !]
$$

There is a simpler expression for $P_{r}(x, 1)=P_{r}(x)$ : 


$$
\begin{aligned}
P_{r}(x)= & \frac{\Gamma(\alpha+\beta+2) 2^{-\alpha-\beta-1}(1-r)}{\Gamma(\alpha+1) \Gamma(\beta+1)(1+r)^{\alpha+\beta+2}} \\
& \cdot{ }_{2} F_{1}\left(\frac{\alpha+\beta+2}{2}, \frac{\alpha+\beta+3}{2} ; \beta+1 ; \frac{2 r(1+x)}{(1+r)^{2}}\right) .
\end{aligned}
$$

See [8, Example 19, p. 102] or it can be derived from a related series computed in $[21,132(10)]$.

One interesting result which follows immediately from (2.5) and Theorem A is the following

Theorem 1. For $\alpha \geqq \beta$, and either $\beta \geqq-\frac{1}{2}$ or $\alpha+\beta \geqq 0$, the Poisson kernel for Jacobi series (2.1) is nonnegative for $r_{0} \leqq r<1$ where $r_{0}$ is the largest zero of

$$
{ }_{2} F_{1}\left(\frac{1}{2}(\alpha+\beta+2), \frac{1}{2}(\alpha+\beta+3) ; \beta+1 ; 4 r /(1+r)^{2}\right)=0 .
$$

This question was posed in an unpublished paper of D. K. McGraw and J. F. Wagner. The corresponding result for the Krawtchouk polynomials was obtained by Eagleson [10]. He also gives a statistical interpretation.

In addition to the case $\alpha=\beta$, which is well known, Theorem 1 can be made more explicit when $\alpha=\beta+1$. Recall

$$
{ }_{2} F_{1}(a, b ; c ; x)=(1-x)^{-a}{ }_{2} F_{1}(a, c-b ; c ; x /(x-1))
$$

This gives

$$
\begin{aligned}
P_{r}(x)= & \frac{\Gamma(\alpha+\beta+2) 2^{-\alpha-\beta-1}}{\Gamma(\alpha+1) \Gamma(\beta+1)} \frac{(1-r)}{\left(1-2 x r+r^{2}\right)^{(\alpha+\beta+2) / 2}} \\
& \cdot{ }_{2} F_{1}\left(\frac{\alpha+\beta+2}{2}, \frac{\beta-\alpha-1}{2} ; \beta+1 ; \frac{-2 r(1+x)}{1-2 x r+r^{2}}\right) .
\end{aligned}
$$

When $x=1, \alpha=\beta+1,(2.8)$ reduces to

$$
A_{2} F_{1}\left(\alpha+\frac{1}{2},-1 ; \alpha ;-4 r(1+r)^{-2}\right)
$$

where $A$ will denote a positive constant or even a positive function whose value does not concern us. Thus (2.6) is

$$
1+4 r_{0}\left(\alpha+\frac{1}{2}\right) / \alpha\left(1-r_{0}\right)^{2}=0
$$

or

$$
r_{0}=\left[-1-\alpha+(1+2 \alpha)^{1 / 2}\right] / \alpha, \quad \alpha \geqq \frac{1}{2} .
$$

For $\alpha=\frac{1}{2}, \beta=-\frac{1}{2}$ the Poisson kernel is positive for $-3+2 \sqrt{ } 2 \leqq r<1$. This special case is interesting since

$$
\frac{P_{n}^{(1 / 2,-1 / 2)}(\cos \theta)}{P_{n}^{(1 / 2,-1 / 2)}(1)}=\frac{\sin \left(n+\frac{1}{2}\right) \theta}{(2 n+1) \sin (\theta / 2)} .
$$


3. Cesàro summability of Jacobi series. The $(C, \gamma)$ means of the formal series $\sum_{n=0}^{\infty} a_{n}$ are defined by

$$
\sigma_{n}^{\gamma}(a)=\frac{n !}{(\gamma+1)_{n}} \sum_{k=0}^{n} \frac{(\gamma+1)_{n-k}}{(n-k) !} a_{k} .
$$

We will only be concerned with positivity properties of $\sigma_{n}^{\gamma}$ and so will consider

$$
\tau_{n}^{\gamma}(a)=\sum_{k=0}^{n} \frac{(\gamma+1)_{n-k}}{(n-k) !} a_{k} .
$$

Clearly $\tau_{n}^{\gamma}(a) \geqq 0$ if and only if $\sigma_{n}^{\gamma}(a) \geqq 0$ when $\gamma>-1$. There is an alternate way to define $\tau_{n}^{\gamma}(a)$. If the $\left\{a_{n}\right\}$ do not grow too rapidly the series $f(r)=\sum_{n=0}^{\infty} a_{n} r^{n}$ converges for some $r>0 . \tau_{n}^{\gamma}(a)$ are given by

$$
(1-r)^{-\gamma-1} f(r)=\sum_{n=0}^{\infty} \tau_{n}^{\gamma}(a) r^{n}
$$

where the series (3.1) converges for some $r>0$.

The positivity of the $(C, \gamma)$ means of the Poisson kernel $P_{n}(x)$ is then determined by the coefficients $g_{n}$ in

$$
\begin{aligned}
& \frac{{ }_{2} F_{1}\left(\frac{1}{2}(\alpha+\beta+2), \frac{1}{2}(\alpha+\beta+3) ; \beta+1 ; 2 r(1+x) /(1+r)^{2}\right)}{(1-r)^{\gamma}(1+r)^{\alpha+\beta+2}} \\
& =\sum_{n=0}^{\infty} g_{n}(x ; \gamma, \alpha, \beta) r^{n} .
\end{aligned}
$$

At present the following results are known about $g_{n}: g_{n}(x ; \gamma, \alpha, \beta) \geqq 0$ if

$$
\begin{aligned}
& \alpha=\beta \geqq-\frac{1}{2}, \quad \gamma=2 \alpha+2, \quad[17],[5] \\
& \alpha=\frac{1}{2}, \quad \beta=-\frac{1}{2}, \quad \gamma=2, \quad[13] .
\end{aligned}
$$

The "correct" state of affairs seems to be

Conjecture 1. If $\alpha \geqq \beta \geqq-\frac{1}{2}, \gamma=\alpha+\beta+2$,

$$
g_{n}(x ; \alpha+\beta+2, \alpha, \beta) \geqq 0, \quad-1 \leqq x \leqq 1 .
$$

For $\alpha=\beta \geqq-\frac{1}{2}$ this conjecture is Kogbetliantz's result (3.3) and for $\alpha=\frac{1}{2}$, $\beta=-\frac{1}{2}$ it is Fejér's result (3.4).

Theorem 2. If Conjecture 1 is true for $(\alpha, \beta)$ then it is true for $(\alpha-\mu, \beta+\mu)$, $0 \leqq \mu \leqq(\alpha-\beta) / 2$.

Theorem 2 follows from the more general

Theorem 3. If

$$
\sum_{n=0}^{\infty} a_{n} P_{n}^{(\alpha, \beta)}(x) / P_{n}^{(\beta, \alpha)}(1) \geqq 0, \quad-1 \leqq x \leqq 1, \alpha>\beta,
$$


then

$$
\begin{aligned}
\sum_{n=0}^{\infty} a_{n} P_{n}^{(\alpha-\mu, \beta+\mu)}(x) / P_{n}^{(\beta+\mu, \alpha-\mu)}(1) \geqq & 0, \\
& -1 \leqq x \leqq 1, \mu>0 ; \alpha-\mu>-1 .
\end{aligned}
$$

Theorem 3 is an immediate consequence of Bateman's integral

$$
\begin{aligned}
&(1+x)^{\beta+\mu} \frac{P_{n}^{(\alpha-\mu, \beta+\mu)}(x)}{P_{n}^{(\beta+\mu, \alpha-\mu)}(1)} \\
&= \frac{\Gamma(\beta+\mu+1)}{\Gamma(\beta+1) \Gamma(\mu)} \int_{-1}^{x}(1+y)^{\beta} \frac{P_{n}^{(\alpha, \beta)}(y)}{P_{n}^{(\beta, \alpha)}(1)}(x-y)^{\mu-1} d y, \\
& \mu>0,-1<x \leqq 1 .
\end{aligned}
$$

See $[4,(3.4)]$.

Theorem 2 in conjunction with (3.4) proves Conjecture 1 for $\alpha=-\beta$, $0 \leqq \alpha \leqq \frac{1}{2}$. To handle other values of $(\alpha, \beta)$ we must look at $g_{n}$ in more detail. Consider the case $\alpha=\beta+1$ where there is some simplification. Using (2.7), (3.2) becomes

$$
\begin{aligned}
&\left(1-r^{2}\right)^{-2 \alpha-1}{ }_{2} F_{1}\left(\alpha+\frac{1}{2}, \alpha+1 ; \alpha ; 2(1+x) r /(1+r)^{2}\right)=\sum_{n=0}^{\infty} g_{n} r^{n} \\
&=(1-r)^{-2 \alpha-1}\left(1-2 x r+r^{2}\right)^{-\alpha-1 / 2} \\
& \cdot{ }_{2} F_{1}\left(\alpha+\frac{1}{2},-1 ; \alpha ;-2(1+x) r /\left(1-2 x r+r^{2}\right)\right) \\
&= {\left[1+\frac{2(1+x) r\left(\alpha+\frac{1}{2}\right)}{\alpha\left(1-2 x r+r^{2}\right)}\right] \frac{1}{\left(1-2 x r+r^{2}\right)^{\alpha+1 / 2}(1-r)^{2 \alpha+1}} } \\
&= \frac{\alpha(1+r)^{2}+r(1+x)}{\alpha\left(1-2 x r+r^{2}\right)^{\alpha+3 / 2}(1-r)^{2 \alpha+1}} .
\end{aligned}
$$

Letting $\alpha=\gamma+\frac{1}{2}$ this reduces to

$$
\frac{\left[\gamma(1+r)^{2}+\frac{1}{2}(1+r)^{2}+r(1+x)\right]}{\left(\gamma+\frac{1}{2}\right)\left[\left(1-2 x r+r^{2}\right)^{2}(1-r)^{2}\right]} \frac{1}{\left[\left(1-2 x r+r^{2}\right)^{1 / 2}(1-r)\right]^{2 \gamma}}
$$

When $\gamma>0$ the function $1 /\left[(1-r)^{2}\left(1-2 x r+r^{2}\right)\right]^{\gamma}$ has positive power series coefficients. This was proven in [5]. The term 


$$
\frac{\frac{1}{2}(1+r)^{2}+r(1+x)}{(1-r)^{2}\left(1-2 x r+r^{2}\right)^{2}}
$$

is the only term left when $\gamma=0$, or $\alpha=\frac{1}{2}$, and so reduces to Fejér's result (3.4), and thus has positive coefficients. The remaining term is

$$
\frac{(1+r)^{2}}{(1-r)^{2}\left(1-2 x r+r^{2}\right)^{2}}=\left[\frac{\left(1-r^{2}\right)}{(1-r)^{2}\left(1-2 x r+r^{2}\right)}\right]^{2} \text {. }
$$

The positivity of the power series coefficients of $\left(1-r^{2}\right) /(1-r)^{2}\left(1-2 x r+r^{2}\right)$ is just Fejér's classical result concerning the $(C, 1)$ summability of Fourier series.

Theorem 2 then gives Conjecture 1 for $(\alpha-\mu, \alpha-1+\mu), 0 \leqq \mu \leqq \frac{1}{2}, \alpha \geqq \frac{1}{2}$. Next set $\gamma=\alpha+\beta+2, \alpha=\beta+2$ in (3.2). A routine calculation using (2.7) gives

$$
\begin{aligned}
& \frac{(1+r)^{3}+(3 /(\alpha-1))(x+1) r(1+r)}{(1-r)^{2 \alpha}\left(1-2 x r+r^{2}\right)^{\alpha+3 / 2}} \\
& =\frac{3}{2(\alpha-1)}\left[\frac{(1+r)^{2}+2(x+1) r}{(1-r)^{2}\left(1-2 x r+r^{2}\right)^{2}}\right] \frac{1+r}{(1-r)^{2 \alpha-2}\left(1-2 x r+r^{2}\right)^{\alpha-1 / 2}} \\
& \quad+\frac{(-3 /(2(\alpha-1))+1)(1+r)^{3}}{(1-r)^{2 \alpha}\left(1-2 x r+r^{2}\right)^{\alpha+3 / 2}} .
\end{aligned}
$$

If $\alpha>1$ the first coefficient is positive. The next factor in the first term is just the kernel Fejér needed for the $(C, 2)$ summability for $\alpha=\frac{1}{2}, \beta=-\frac{1}{2}$, and so it has positive coefficients. The remaining factor is

$$
\frac{1-r^{2}}{(1-r)^{2 \alpha-1}\left(1-2 x r+r^{2}\right)^{\alpha-1 / 2}}
$$

and if $\alpha \geqq \frac{3}{2}$ this has positive coefficients by Kogbetliantz's result (3.3).

The second term is

$$
\begin{gathered}
\left(\frac{2 \alpha-5}{2 \alpha-2}\right)\left[\frac{\left(1-r^{2}\right)^{3}}{(1-r)^{2 \alpha+3}\left(1-2 x r+r^{2}\right)^{\alpha+3 / 2}}\right] \\
=\left(\frac{2 \alpha-5}{2 \alpha-2}\right)\left[\frac{1-r^{2}}{(1-r)^{2}\left(1-2 x r+r^{2}\right)}\right]^{2} \\
\cdot \frac{1-r^{2}}{(1-r)^{2 \alpha-1}\left(1-2 x r+r^{2}\right)^{\alpha-1 / 2}}
\end{gathered}
$$

and if $\alpha \geqq \frac{5}{2}$ the first factor is nonnegative, and the remaining two factors have positive coefficients if $\alpha \geqq \frac{3}{2}$. Thus the $(C, \alpha+\beta+2)$ means are positive for $\alpha=\beta+2, \alpha \geqq \frac{5}{2}$. Applying Theorem 2 again gives positive $(C, \alpha+\beta+2)$ means when $\beta+1 \leqq \alpha \leqq \beta+2, \beta \geqq \frac{1}{2}$, and $\alpha+\beta \geqq 3$ hold.

These results can be summarized in 
Theorem 4. The $(C, \alpha+\beta+2)$ means of (2.1) are positive for

(i) $\alpha=\beta \geqq-\frac{1}{2}$,

(ii) $\alpha+\beta=2 c, c \geqq 0, c-\frac{1}{2} \leqq \alpha \leqq c+\frac{1}{2}$,

(iii) $\alpha+\beta=2 c, c \geqq \frac{3}{2}, c-1 \leqq \alpha \leqq c+1$.

In $\$ 5$ we will show that these results are best possible, in the sense that the $(C$, $\gamma)$ means are not positive for $\gamma<\alpha+\beta+2$.

The main application of positivity at present is to the Lagrange interpolation problem, and for this problem all that is needed is some positive Cesàro mean. This can be obtained for most $(\alpha, \beta)$ in the following way.

Let $\alpha=\beta+k, k=1,2,3, \ldots$ Recall that $g_{n}=g_{n}(x ; \gamma, \alpha, \beta)$ is given by

$$
\begin{aligned}
& (1-r)^{-\gamma}(1+r)^{-\alpha-\beta-2}{ }_{2} F_{1}\left(\frac{\alpha+\beta+3}{2}, \frac{\alpha+\beta+2}{2} ; \beta+1 ; \frac{2 r(1+x)}{(1+r)^{2}}\right) \\
& =\sum_{n=0}^{\infty} g_{n} r^{n} .
\end{aligned}
$$

If $k$ is even then (3.2) becomes

$$
\begin{aligned}
(1-r)^{-\gamma}(1-r)^{-\alpha-\beta-2}\left(1-\frac{2 r(1+x)}{(1+r)^{2}}\right)^{-(\alpha+\beta+3) / 2} \\
\quad \cdot{ }_{2} F_{1}\left(\frac{\alpha+\beta+3}{2},-\frac{k}{2} ; \beta+1 ; \frac{-2 r(1+x)}{1-2 x r+r^{2}}\right) \\
=\frac{1+r}{(1-r)^{\gamma}} \sum_{j=0}^{k / 2} \frac{\left(\frac{1}{2}(\alpha+\beta+3)\right)_{j}\left(-\frac{1}{2} k\right)_{j}(-1)^{j}}{(\beta+1)_{j} j !} \frac{[2 r(1+x)]^{j}}{\left(1-2 x r+r^{2}\right)^{j+(\alpha+\beta+3) / 2}} \\
=\frac{1+r}{(1-r)^{\gamma}} \sum_{j=0}^{k / 2} \frac{\left(\frac{1}{2}(\alpha+\beta+3)\right)_{j}\left(\frac{1}{2} k\right)\left(\frac{1}{2} k-1\right) \ldots\left(\frac{1}{2} k-j+1\right) 2^{j}(1+x)^{j} r^{j}}{(\beta+1)_{j} j !\left(1-2 x r+r^{2}\right)^{j+(\alpha+\beta+3) / 2}} .
\end{aligned}
$$

A similar expression is obtained when $k$ is odd.

If $\gamma$ is sufficiently large

$$
\frac{1}{(1-r)^{\gamma}\left(1-2 x r+r^{2}\right)^{j+(\alpha+\beta+3) / 2}}
$$

has positive power series coefficients [5], since $(\alpha+\beta+3) / 2 \geqq 0$.

All of the coefficients in (3.5) are positive so $g_{n}$ is positive. Then applying Theorem 2 we obtain

Theorem 5. The Cesàro means of some finite order of (2.1) are positive if

(i) $\alpha, \beta \geqq-\frac{1}{2}$,

(ii) $-1<\beta<-\frac{1}{2},|\alpha-k| \leqq 1+\beta, k=1,2, \ldots$,

(iii) $-1<\beta<-\frac{1}{2},\left|\alpha-k-\frac{1}{2}\right| \leqq 1+\beta, k=1,2, \ldots$,

(iv) the symmetric regions to (ii) and (iii) when $\alpha$ and $\beta$ are interchanged.

For each of these values of $(\alpha, \beta)$ a Cesàro mean which is positive could be determined. However the only interest in finding a specific mean is when the best possible mean has been found. 
4. Hankel transforms. One way of attempting to find out if Conjecture 1 is true is to examine some of its consequences. Some will be dealt with in \$5. One important corollary deals with the Cesàro summability of Hankel transforms. The Poisson kernel for Hankel transforms is

$$
\int_{0}^{\infty} e^{-\varepsilon t} J_{\alpha}(x t) J_{\alpha}(y t) t d t
$$

Using

$$
J_{\alpha}(t)=(t / 2)^{\alpha} 1 / \Gamma(\alpha+1)+\ldots,
$$

we see that the positivity of $(4.1)$ (which holds $[25,13.22(1)])$ implies

$$
\int_{0}^{\infty} e^{-\varepsilon t} J_{\alpha}(x t) t^{\alpha+1} d t \geqq 0 .
$$

Conversely, using the positivity of the generalized translation operator the positivity of (4.3) implies that of (4.1), even when $e^{-\varepsilon t}$ is replaced by a general function $f(t)$. The positivity of the $(C, \gamma)$ means of $(4.3)$ is equivalent to

$$
\int_{0}^{x}(x-t)^{\gamma} t^{\alpha+1} J_{\alpha}(t) d t \geqq 0 .
$$

The connection between Jacobi polynomials and Bessel functions comes from

$$
\lim _{n \rightarrow \infty} n^{-\alpha} P_{n}^{(\alpha, \beta)}(\cos \theta / n)=(\theta / 2)^{-\alpha} J_{\alpha}(\theta)
$$

Szegö [24] observed that the positivity of certain Jacobi sums implies the positivity of certain Bessel function integrals when (4.5) is used. We will list the consequences of the conjectures and results of $\$ 3$.

\section{Theorem 6.}

$$
\int_{0}^{x}(x-t)^{2 \alpha+2} t^{\alpha+1} J_{\alpha}(t) d t \geqq 0, \quad \alpha \geqq-\frac{1}{2}
$$

This follows Kogbetliantz's result (3.3).

\section{Conjecture 2.}

$$
\int_{0}^{x}(x-t)^{\alpha+3 / 2} t^{\alpha+1} J_{\alpha}(t) d t \geqq 0, \quad \alpha \geqq-\frac{1}{2} .
$$

This is the limiting form of Conjecture 1 for $\beta=-\frac{1}{2}$. While we only know Conjecture 1 for $\alpha=-\frac{1}{2}$ and $\alpha=\frac{1}{2}$ when $\beta=-\frac{1}{2}$, Conjecture 2 is true for $\alpha=k-\frac{1}{2}, k=0,1, \ldots$ This will be proven below when Conjecture 2 is translated into another problem.

Theorem 7.

$$
\int_{0}^{x}(x-t)^{2 \alpha} t^{\alpha} J_{\alpha}(t) d t \geqq 0, \quad \alpha \geqq-\frac{1}{2}
$$


Theorem 7 will be proven below. For $\alpha \geqq \frac{1}{2}$, a stronger result than Theorem 7 follows from Theorem 6 . For $0<\alpha<\frac{1}{2}$ a stronger result is also true.

$$
\int_{0}^{x} t^{\alpha} J_{\alpha}(t) d t \geqq 0
$$

follows from

$$
\sum_{k=0}^{n} \frac{P_{k}^{(\alpha,-\alpha)}(x)}{P_{k}^{(-\alpha, \alpha)}(1)} \geqq 0, \quad-1 \leqq x \leqq 1,0 \leqq \alpha \leqq \frac{1}{2}
$$

For $\alpha=0$ this is the same as Theorem 7, which is an old result of Cooke [9]. This result of Cooke has inspired a number of beautiful theorems [19], [20], [23].

One method of attacking these problems is to take Laplace transforms. Recall that

$$
\begin{gathered}
\int_{0}^{\infty} e^{-x t} t^{\alpha} d t=\frac{\Gamma(\alpha+1)}{x^{\alpha+1}}, \\
\int_{0}^{\infty} t^{\alpha+1} J_{\alpha}(t) e^{-x t} d t=\frac{2^{\alpha+1} x \Gamma(\alpha+3 / 2)}{\left(x^{2}+1\right)^{\alpha+3 / 2} \Gamma\left(\frac{1}{2}\right)} \quad[25,13.2(6)], \\
\int_{0}^{\infty} t^{\alpha} J_{\alpha}(t) e^{-x t} d t=\frac{2^{\alpha} \Gamma\left(\alpha+\frac{1}{2}\right)}{\left(x^{2}+1\right)^{\alpha+1 / 2} \Gamma\left(\frac{1}{2}\right)} \quad[25,13.2(5)] .
\end{gathered}
$$

A function $f(x)$ is completely monotonic on $(0, \infty)$ if $f(x)=\int_{0}^{\infty} e^{-x t} d \mu(t), d \mu(t)$ a nonnegative Borel measure, and the integral converges for $0<x<\infty$.

Conjecture 2 is equivalent to

Conjecture 2'. 1/ $x^{c}\left(x^{2}+1\right)^{c}$ is completely monotonic for $c \geqq 1$.

Conjecture $2^{\prime}$ holds for $c=1,2, \ldots$, since

$$
\frac{1}{x\left(x^{2}+1\right)}=\int_{0}^{\infty}(1-\cos t) e^{-x t} d t
$$

and the product of completely monotonic functions is completely monotonic, so Conjecture 2 holds for $\alpha=-\frac{1}{2}, \frac{1}{2}, \ldots$

Theorem 7 is equivalent to

Theorem B. $1 / x^{2 c}\left(x^{2}+1\right)^{c}$ is completely monotonic for $c>0$.

This was proven in [5].

Theorem 6 is equivalent to

Theorem 6'. $1 / x^{2 c-1}\left(x^{2}+1\right)^{c}$ is completely monotonic for $c \geqq 1$.

These problems should be easier to do than the corresponding problems for Jacobi series. However I do not know how to prove Conjecture $2^{\prime}$ for other values of $c$. 
The known cases of Conjecture $2^{\prime}$ and (4.6) can be used to improve Theorem $6^{\prime}$ for $c \geqq \frac{1}{2}$.

\section{Theorem 8.}

$$
\frac{1}{x^{d}\left(x^{2}+1\right)^{c}} \frac{1}{x^{k}\left(x^{2}+1\right)^{k}}
$$

is completely monotonic for $d=2 c, 0<c \leqq \frac{1}{2} ; d=1, \frac{1}{2} \leqq c<1 ; k=0,1, \ldots$

This suggests the interesting problem of determining when $x^{-d}\left(x^{2}+1\right)^{-c}$ is a completely monotonic function. I have been careful not to give Conjecture 1 for $-1<\beta<-\frac{1}{2}, \alpha+\beta \geqq 0$. This may have been conservatism on my part, and if it is true then Conjecture 2 could be improved for $c$ large. At present I am unwilling to conjecture that $d$ could ever be less than $c$ in the above problem. Not only are we lacking methods to prove these conjectures, we also have no methods to show they are best possible. The methods to be used in the next section to show that Conjecture 1 cannot be improved for fixed $(\alpha, \beta)$ are not available in this case.

It is interesting to observe the difference between the Cesàro means of (4.3) and the Riesz means. When $\alpha \geqq-\frac{1}{2}$ the Cesàro means of a sufficiently high order are positive. This is not true of the Riesz means, since

$$
x^{\alpha+\gamma} J_{\alpha+\gamma}(x)=\frac{1}{2^{\alpha} \Gamma(\alpha+1)} \int_{0}^{x}\left(x^{2}-y^{2}\right)^{\gamma} t^{\alpha+1} J_{\alpha}(t) d t \quad[25,12.11(1)],
$$

and $J_{\alpha+\gamma}(x)$ changes sign infinitely of ten for every $\gamma$.

5. Applications. For some problems it is essential to look at the partial sums, and yet a positive kernel is needed. Clearly this cannot be done even for Fourier series, but de la Vallée Poussin showed how to use the $(C, 1)$ means to construct the partial sums at the expense of leaving a tail. If $\sigma_{n-1}=\sum_{k=0}^{n} a_{k}(1-k / n)$ then

$$
2 \sigma_{2 n-1}-\sigma_{n-1}=\sum_{k=0}^{n} a_{k}+2 \sum_{k=n+1}^{2 n-1} a_{k}(1-k /(2 n)) .
$$

Zygmund [26] has shown how to obtain a similar result for $(C, 2)$ means and Stein [22] has handled the case of $(C, k)$ means, $k=1,2, \ldots$ We will give a simple modification of Stein's proof to prove

Theorem 9. Let $k$ be a positive integer. There exist $k+1$ real parameters $\alpha_{j}^{k}$, $j=1, \ldots, k+1$, which are uniformly bounded,i.e. $\left|\alpha_{j}^{k}\right| \leqq A$, where $\alpha_{j}^{k}$ depends on $n$ but $A$ is independent of $n$. Then there is an $N$ and numbers $\lambda_{k, n}$ so that

$$
\tau_{n}^{k}=\alpha_{1}^{k} \sigma_{n-1}^{k}+\alpha_{2}^{k} \sigma_{n+[n / k]-1}^{k}+\ldots+\alpha_{k+1}^{k} \sigma_{2 n-1}^{k}
$$

and

$$
\tau_{n}^{k}(a)=\sum_{k=0}^{n-1} a_{k}+\sum_{k=n}^{2 n-1} \lambda_{k, n} a_{k}, \quad n \geqq N
$$


The case $k=2$ is typical.

$$
\begin{aligned}
& \tau_{2}=\alpha_{1} \sigma_{n-1}+\alpha_{2} \sigma_{[3 n / 2]-1}+\alpha_{3} \sigma_{2 n-1} \\
& \begin{aligned}
=\sum a_{k}\left\{\alpha_{1}(1-\right. & k / n)(1-k /(n+1)) \\
& +\alpha_{2}(1-k /([3 n / 2])(1-k /([(3 n / 2)]+1))) \\
& \left.+\alpha_{3}(1-k /(2 n))(1-k /(2 n+1))\right\}
\end{aligned}
\end{aligned}
$$

The only change in Stein's proof is that the Vandermonde determinant is now

$$
\left|\begin{array}{ccc}
1 & 1 & 1 \\
1 & \frac{2}{3} & \frac{1}{2} \\
1 & \left(\frac{2}{3}\right)^{2} & \left(\frac{1}{2}\right)^{2}
\end{array}\right|
$$

and this does not vanish.

Combining Theorem 9 with Theorem 5 and the proof in [1] we obtain

Theorem 10. Let $L_{n}^{f}(x)$ be the Lagrange interpolation polynomial at the zeros of $P_{n}^{(\alpha, \beta)}(x), \alpha \geqq \beta$. If $f$ is continuous,

$$
\lim _{n \rightarrow \infty} \int_{-1}^{1}\left|L_{n}^{f}(x)-f(x)\right|^{p}(1-x)^{\alpha}(1+x)^{\beta} d x=0
$$

for $p<4(\alpha+1) /(2 \alpha+1)$ when

(i) $\alpha, \beta \geqq-\frac{1}{2}$,

(ii) $|\alpha-k| \leqq 1+\beta,-1<\beta<-\frac{1}{2}, 2 k=2,3, \ldots$.

To show that Conjecture 1 cannot be improved we prove

Theorem 11. Let $g(r)$ have positive Cesàro means of order $\gamma>0$.

$$
\begin{aligned}
g(r) & =\sum_{n=0}^{\infty} c_{n} r^{n}, \\
(1-r)^{-\gamma-1} g(r) & =\sum_{n=0}^{\infty} S_{n}^{\gamma} r^{n}, \quad S_{n}^{\gamma} \geqq 0, n=0,1, \ldots
\end{aligned}
$$

Then $g_{n}(r)=\sum_{k=0}^{n} c_{k} r^{k} \geqq 0,0 \leqq r \leqq 1 /(\gamma+1)$.

Fejér [12, Satz VI] has proven Theorem 10 for $\gamma$ an integer.

Let $g_{n}(r)=\sum_{k=0}^{n} c_{k} r^{k}$. Then

$$
\begin{aligned}
g_{n}(r) & =(1-r)^{\gamma+1} g_{n}(r)(1-r)^{-(\gamma+1)}=\sum_{k=0}^{n} S_{k, n} r^{k} \sum_{j=0}^{\infty}(-\gamma-1)_{j} r^{j} / j ! \\
& =\sum_{k=0}^{n} S_{k, n} r^{k} \sum_{j=0}^{n-k}(-\gamma-1)_{j} r^{j} / j !
\end{aligned}
$$


since $g_{n}(r)$ is a polynomial of degree $n$ and so all of the terms which were dropped could be summed to zero. But $S_{k, n}$, which was formed from $c_{0}, c_{1}, \ldots, c_{n}$, $0,0, \ldots$, only uses $c_{0}, \ldots, c_{k}$ since $k \leqq n$, so $S_{k, n}=S_{k} \geqq 0$. Thus $g_{n}(r) \geqq 0$ if

$$
\sum_{j=0}^{n-k} \frac{(-\gamma-1)_{j} r^{j}}{j !} \geqq 0
$$

For $k=n-1$ this is $1-(\gamma+1) r \geqq 0$, so $r \leqq 1 /(\gamma+1)$ is a necessary condition. It is also sufficient. The successive terms decrease in absolute value when $j$ increases for $r \leqq 1 /(\gamma+1)$, since

$$
\left|\frac{(-\gamma-1+j) r}{j+1}\right| \leqq \frac{|j-\gamma-1|}{(j+1)(\gamma+1)}<1, \quad j=1,2, \ldots, \gamma>0
$$

The terms also alternate in sign, except when $\gamma$ is not an integer when there is one exception. The worst case comes when $1<\gamma<2$, when two adjacent negative terms occur for small $j$. In this case we have

$$
\begin{gathered}
1-(\gamma+1) r+\frac{(\gamma+1) \gamma r^{2}}{2 !}-\frac{(\gamma+1)(\gamma)(\gamma-1) r^{3}}{3 !} \\
+\frac{(\gamma+1)(\gamma)(\gamma-1)(\gamma-2) r^{4}}{4 !}
\end{gathered}
$$

The sum of the last three terms is

$$
\begin{aligned}
& \frac{(\gamma+1)(\gamma) r^{2}}{2}\left[1-\frac{(\gamma-1) r}{3}+\frac{(\gamma-1)(\gamma-2)}{3 \cdot 4} r^{2}\right] \\
& \geqq \frac{(\gamma+1) \gamma r^{2}}{4 !(\gamma+1)^{2}}\left[12(\gamma+1)^{2}-4\left(\gamma^{2}-1\right)+(\gamma-1)(\gamma-2)\right] \\
& \geqq \frac{(\gamma+1) \gamma r^{2}}{4 !(\gamma+1)^{2}}[48-12-2]>0 .
\end{aligned}
$$

The other cases are even easier.

If Conjecture 1 could be improved so that the $(C, \gamma)$ means of $(2.1)$ were positive for some $\gamma<\alpha+\beta+2$, then

$$
\sum_{k=0}^{n} r^{k} \frac{(2 k+\alpha+\beta+1) \Gamma(k+\alpha+\beta+1)}{\Gamma(k+\beta+1)} P_{k}^{(\alpha, \beta)}(x) \geqq 0
$$

would hold for $r \leqq 1 /(\gamma+1)$. But (5.1) can only hold for $r \leqq 1 /(\alpha+\beta+3)$ when $n=1, x=-1$. See [2]. This method is not available for Hankel transforms, because the point which would correspond to $x=-1$ is at infinity.

We also see that Conjecture 1 cannot $^{\circ}$ be improved by setting $x=-1$ in (3.2). Then $g_{1}$ is negative if $\gamma<\alpha+\beta+2$. 


\section{BIBLIOGRAPHY}

1. R. Askey, Mean convergence of orthogonal series and Lagrange interpolation, Acta Math. 23 (1972), 71-85.

2.—_ Nonnegative sums of Jacobi polynomials, Tôhoku Math. J. (2) 24 (1972), 109-119.

3. R. Askey and J. Fitch, Positivity of the Cotes numbers for some ultraspherical abscissas, SIAM J. Numer. Anal. 5 (1968), 199-201. MR 37 \#3750.

4._, Integral representations for Jacobi polynomials and some applications, J. Math. Anal. Appl. 26 (1969), 411-437. MR 38 \#6128.

5. R. Askey and H. Pollard, Some absolutely monotonic and completely monotonic functions, SIAM J. Math. Anal. 4 (1973).

6. R. Askey and S. Wainger, A convolution structure for Jacobi series, Amer. J. Math. 91 (1969), 463-485. MR 41 \#8728.

7. W. N. Bailey, The generating function of Jacobi polynomials, J. London Math. Soc. 13 (1938), 8-12.

8.—, Hypergeometric functions, Cambridge Univ. Press, Cambridge, 1935.

9. R. G. Cooke, A monotonic property of Bessel functions, J. London Math. Soc. 12 (1937), 180-185.

10. G. K. Eagleson, A characterization theorem for positive definite sequences on the Krawtchouk polynomials, Austral. J. Statist. 11 (1969), 29-38.

11. L. Fejér, Sur les fonctions bornées et intégrables, C. R. Acad. Sci. Paris 131 (1900), 984-987; Gesammelte Arbeiten I, 37-41.

12.—- Einige Sätze, die sich aug das Vorzeichen einer ganzen rationalen Funktion beziehen usw., Monatsh. Math. 35 (1928), 305-344; Gesammelte Arbeiten II, 202-237.

13.—, Neue Eigenschaften der Mittelwerte bei den Fourierreihen, J. London Math. Soc. 8 (1933),

53-62; Gesammelte Arbeiten II, 493-501.

14. E. Feldheim, On the positivity of certain sums of ultraspherical polynomials, J. Analyse Math. 11 (1963), 275-284. MR 28 \#1333.

15. G. Gasper, Positivity and convolution structure for Jacobi series, Ann. of Math. (2) 93 (1971), $112-118$.

16. - Banach algebras for Jacobi series and positivity of a kernel, Ann. of Math. (2) 95 (1972), 261-280.

17. E. Kogbetliantz, Recherches sur la sommabilité des séries ultersphériques par la méthode des moyennes arithmétiques, J. Math. Pures Appl. (9) 3 (1924), 107-187.

18. T. Koornwinder, Jacobi polynomials. II. An analytic proof of the product formula, SIAM J. Math. Anal. 4 (1973).

19. L. Lorch, M. E. Muldoon and P. Szego, Higher monotonicity properties of certain Sturm-Liouville functions. III, Canad. J. Math. 22 (1970), 1238-1265. MR 43 \#603

20. E. Makai, On a monotonic property of certain Sturm-Liouville functions, Acta Math. Acad. Sci. Hungar. 3 (1952), 165-172. MR 14, 872.

21. E. D. Rainville, Special functions, Macmillan, New York, 1960. MR 21 \#6447.

22. E. M. Stein, Interpolation in polynomial classes and Markoff s inequality, Duke Math. J. 24 (1957), 467-476. MR 19, 956.

23. J. Steinig, On a monotonicity property of Bessel functions, Math. Z. 122 (1971), 363-365.

24. G. Szegö, Orthogonal polynomials, Amer. Math. Soc. Colloq. Publ., vol. 23, Amer. Math. Soc., Providence, R.I., 1967.

25. G. N. Watson, $A$ treatise on the theory of Bessel functions, Cambridge Univ. Press, Cambridge, 1944. MR 6, 64.

26. A. Zygmund, A property of the zeros of Legendre polynomials, Trans. Amer. Math. Soc. 54 (1943), 39-56. MR 5, 180.

Department of Mathematics, University of Wisconsin, Madison, Wisconsin 53706 